$>$ Le séquençage à haut débit est en train de révolutionner les différents diagnostics génétiques et notamment le diagnostic préimplantatoire (DPI), ouvrant les portes à une médecine prédictive de plus en plus performante. Le DPI n'est pas lui-même nouveau, et les différentes législations s'en sont saisies depuis une vingtaine d'années; les comités nationaux d'éthique ont de leur côté largement débattu des tests anténataux dans le cadre de l'assistance médicale à la procréation. Cet article examine les travaux de ces comités concernant le DPI dans trois États, la France, la Belgique et la Suisse, afin de mettre en lumière leurs similitudes et leurs différences. Le sujet a par ailleurs été soulevé et discuté dans le cadre des États généraux de la bioéthique qui se sont déroulés cette année en France et, vu les progrès diagnostiques réalisés depuis peu, ils se sont fait l'écho de certaines demandes de la société civile. Toutefois, en France, c'est la prudence qui continue de dominer. <

Médecine personnalisée, de précision, génomique, prédictive ou préventive, ce sont là les multiples expressions utilisées pour caractériser la nouvelle orientation que prend la médecine de nos jours, tendue vers ce que certains appellent «la santé digitalisée » [1]. Un des points forts de cette nouvelle orientation est l'accent mis sur la prédiction, en particulier à partir de l'analyse du génome des individus, rendue possible par les nouvelles techniques de séquençage à haut débit.

Le diagnostic préimplantatoire (DPI) est un test qui est utilisé depuis les années 1990, soit bien avant l'avènement de la médecine personnalisée. II permet de détecter in vitro des anomalies génétiques et chromosomiques de l'embryon, et d'écarter ceux qui en sont atteints (jusque là, seul un diagnostic prénatal in vivo était disponible) [2]. Les analyses génétiques devenant plus rapides, moins onéreuses et plus faciles, la question se pose naturellement de savoir quelles implications cela peut et doit avoir sur la pratique du DPI : faut-il (doit-on) élargir les indications, multiplier les fécondations in vitro (FIV) pour permettre plus de tests ou, au contraire, les limiter, voire les interdire, pour éviter toute dérive et toute pente glissante?

C'est l'un des thèmes qui ont été abordés lors des États généraux de la bioéthique cette année en France. Comme on le lit dans le Rapport de synthèse du Comité consultatif national d'éthique en sciences de la vie

Vignette (Photo @ Inserm - Patrick Delapierre).

\section{Le diagnostic préimplantatoire (DPI) à l'ère de la médecine prédictive}

Bernard Baertschi

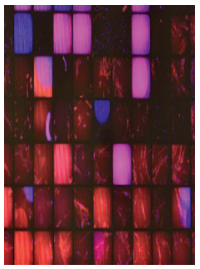

Institut Éthique, Histoire, Humanités (iદH2), université de Genève, CMU, 1 rue MichelServet, CP 1211 Genève 4, Suisse.

bernard.baertschi@unige.ch

et de la santé (CCNE), qui consacre un chapitre entier aux examens génétiques («Examens génétiques et médecine génomique ») : «L'approche de ces diagnostics prénatals (DPN, DPI), mais aussi en postnatal, est bouleversée par l'avènement de technologies nouvelles de séquençage et de génétique moléculaire, permettant des études sur des panels de gènes, voire le génome entier, ou la détection d'anomalies chromosomiques fœtales dans le sang maternel, ouvrant ainsi la voie à une extension considérable des indications de l'examen des caractéristiques génétiques à visée diagnostique, mais aussi de dépistage, et ce, hors du contexte des indications ciblées qu'autorise la loi actuellement » [3]. C'est le sujet du DPI que je souhaite étudier. À cet effet et après avoir rappelé ce que dit la loi en France, j'examinerai en un premier temps les avis que le CCNE a émis depuis une vingtaine d'années, afin de mettre en lumière les enjeux éthiques et les arguments discutés. Je mettrai le débat français en perspective, en regardant la manière dont les discussions s'engagent dans deux autres pays voisins et en partie francophones, la Belgique et la Suisse, à propos des tests génétiques. En un second temps, je me demanderai, à la lumière des progrès récents dans le séquençage du génome et des conclusions des États généraux, si, et sur quels points, il serait judicieux de rediscuter la réglementation en vigueur.

\section{Du droit à l'éthique}

La loi française autorise le DPI mais sous des conditions très strictes, comme le souligne l'étude réalisée 
par le Sénat, car «il entraîne une sélection des embryons » [4]. Les conditions de la loi sont les suivantes: "Le code de la santé publique autorise le diagnostic préimplantatoire "à titre exceptionnel" Iorsque "le couple, du fait de sa situation familiale, a une forte probabilité de donner naissance à un enfant atteint d'une maladie génétique d'une particulière gravité reconnue comme incurable au moment du diagnostic". La réalisation du diagnostic préimplantatoire est subordonnée à l'identification préalable "chez l'un des parents ou l'un de ses ascendants immédiats dans le cas d'une maladie gravement invalidante, à révélation tardive et mettant prématurément en jeu le pronostic vital, [de] l'anomalie ou [d]es anomalies responsables d'une telle maladie". En outre, le diagnostic préimplantatoire ne peut avoir pour objet que la recherche de l'affection considérée et les moyens de la prévenir ou de la traiter ».

Cela signifie donc qu'on ne peut mener un test que pour une pathologie précise : la plupart du temps, lorsqu'un premier enfant en a déjà souffert, soit dans une intention thérapeutique si la maladie est «actionnable ${ }^{1}$, soit - et c'est le cas habituel - pour écarter les embryons porteurs de la modification testée qui ne seront alors pas transférés dans l'utérus.

La même Étude du Sénat compare la situation française à celle d'autres pays, notamment la Belgique et la Suisse. On y lit que la Belgique autorise le DPI à des conditions proches de celles qui prévalent en France (loi du 6 juillet 2007), alors que la Suisse l'interdit explicitement (loi du 18 décembre 1998, qui stipule sans ambiguïté : «Le prélèvement d'une ou plusieurs cellules sur un embryon in vitro et leur analyse sont interdits »). À l'instar de la loi française, la loi belge prohibe encore expressément «la sélection à visée eugénique et la sélection exclusivement fondée sur le sexe de l'enfant à naître ». L'Étude du Sénat mentionne aussi que le débat continue en Suisse et que la loi pourrait changer. C'est désormais chose faite. En effet, le 5 juin 2016, le peuple suisse a accepté, lors d'une votation², le DPI pour des situations bien précises, comme on le lit sur le site du Conseil fédéral $^{3}$ : «La loi n'admet l'analyse génétique d'embryons conçus artificiellement que dans deux cas : d'une part, pour les couples porteurs d'une maladie héréditaire grave, d'autre part, pour les couples qui ne peuvent pas procréer par voie naturelle. Toute autre application du DPI demeure interdite » [5]. La procréation médicalement assistée (PMA) (désignée également sous le terme de «Assistance médicale à la procréation » $[\mathrm{AMP}]$ ) a deux finalités : permettre à des parents d'avoir un enfant «à eux » et d'avoir, si possible, un enfant en bonne santé, finalités auxquelles le DPI peut contribuer, mais exclusivement dans ces cas. À la différence du droit français, ni le droit belge ni le droit suisse ne limitent le diagnostic à une seule pathologie, génétique ou chromosomique.

Voilà donc pour le droit. Mais le processus législatif est partout précédé de débats publics et d'interventions d'experts. Parmi les participants à ces débats, certains jouent un rôle particulièrement

\footnotetext{
${ }^{1}$ Sur laquelle une intervention est possible.

${ }^{2}$ Référendum populaire permettant à la population d'exprimer son choix.

${ }^{3}$ L'organe exécutif de la confédération suisse.
}

important: les comités d'éthique. S'ils représentent une éthique institutionnelle, leur liberté de parole est grande, car ils n'ont qu'un rôle consultatif dans les trois pays concernés ici. Plusieurs de leurs avis (France et Belgique) et prises de positions (Suisse) concernent le DPI. Que lit-on dans ces avis et prises de position?

En France, le DPI a fait l'objet de deux avis du CCNE : Réflexions sur l'extension du diagnostic préimplantatoire (avis $n^{\circ} 72$ ) en juillet 2002 [6], et Avis sur les problèmes éthiques liés aux diagnostics anténatals (avis $n^{\circ}$ 107) en octobre 2009 [7]. Tout dernièrement encore, dans son avis $n^{\circ} 129$, Contribution du Comité consultatif national d'éthique à la révision de la loi de bioéthique 2018-2019 [8], le CCNE a consacré quelques développements à la question. L'avis $n^{\circ} 72$ [6] est une réponse à deux saisines concernant un élargissement des indications légales du DPI. La loi française considère en effet exclusivement le bien de l'enfant, et non celui des parents ou de la famille: il s'agit d'éviter la naissance d'un enfant dont la vie sera une souffrance insupportable pour lui. Or, la première saisine concerne le cas de la conception d'un enfant dont le sang, tiré du cordon ombilical, serait utilisé afin de soigner un frère ou une sœur aîné(e) gravement malade. C'est ce que l'on nomme le «bébé-médicament » ou l'« enfant du double espoir », à propos duquel le CCNE pose cette question: «Si l'extension des indications génétiques du DPI pour l'enfant lui-même ne pose pas de problème juridique ou éthique en soi, elle soulève des questions juridiques et éthiques majeures lorsqu'elle concerne l'intérêt d'un tiers » [6]. En effet, un enfant ne doit pas être instrumentalisé à des fins qui ne sont pas les siennes, aussi bonnes soient-elles. Le CCNE penche toutefois en faveur de l'extension de l'autorisation du DPI dans le cas examiné, car il n'y a pas ici d'instrumentalisation proprement dite: «L'enfant naîtra pour lui-même. Il aura sa vie à lui. II ne sera pas devenu uniquement un moyen » [6] (voir également [9]). Le législateur a suivi cette proposition.

Le second avis du CCNE est postérieur à la loi de 2004. II se veut une réflexion plus générale sur les diagnostics anténatals. Concernant le DPI, le CCNE observe deux attitudes dans la société française: "Certains insistent sur la contrepartie négative, qui est l'élimination des embryons porteurs d'une maladie génétique lors de la procédure de tri ; d'autres mettent davantage l'accent sur la souffrance liée à I'IMG ${ }^{4}$ que le DPI peut permettre d'éviter» [7]. II souligne que cette différence vient du statut qui est accordé à l'embryon : pour les uns, l'embryon a la même dignité qu'une personne

\footnotetext{
${ }^{4}$ Interruption médicale de grossesse.
} 
(il est une personne proprement dite), ce qui exclut qu'on puisse le détruire intentionnellement (ce serait alors un meurtre); alors que pour les autres, c'est la question de la qualité de la vie qui compte, celle de l'enfant d'abord, mais aussi celle de sa famille.

Le CCNE souligne fortement l'importance de la finalité du DPI, à savoir éviter une maladie grave à un futur enfant, et donc les souffrances qui y sont liées: «La seule finalité éthique incontestable du DPI est celle de permettre à des couples d'avoir un enfant, alors que leur passé familial ou le handicap sévère d'un premier-né les aurait conduits à y renoncer » [7]. Dans la foulée, il écarte explicitement d'autres finalités : le DPI ne peut avoir ni de but préventif, du moins prioritairement (il ne s'agit pas d'une mesure de santé publique), ni de but eugéniste, ce que la loi condamnerait. Toutefois, «le sens du terme eugénisme retenu par le législateur est celui d'un programme politique, hygiéniste et idéologique, visant à améliorer l'espèce humaine ». Et dans le DPI, il ne s'agit pas de cela: «Les professionnels de santé ne sont pas astreints par des directives imposées par les pouvoirs publics incitant les femmes à recourir à une IMG ou à un tri embryonnaire, lorsqu'elles ont une forte probabilité de donner naissance à un enfant atteint d'une affection grave et incurable » [7]. II reste que si l'État n'exerce ici aucune contrainte, la pression sociale peut se révéler pernicieuse. Un moyen de la contrer est d'améliorer la prise en charge des handicapés, comme cela a été le cas pour le syndrome de Down (ou trisomie $21)^{5}$ : «Au cours des dernières années, la trisomie a fait l'objet d'une prise en charge qui a augmenté la qualité de vie des personnes qui en sont affectées »[7].

On reconnaît ici ce qui est parfois appelé «l'argument expressiviste » (expressivist argument) : la pratique du DPI, et des tests anténatals en général, exprimerait (express) une attitude stigmatisante, non seulement du handicap, mais aussi des handicapés, dont la vie serait perçue comme ayant moins de valeur [10]. Le CCNE souligne, avec raison, que le remède à cette dérive n'est pas l'interdiction des tests - dont la finalité est justement de faire diminuer les souffrances -, mais une meilleure prise en charge.

À la fin de son avis de 2009, le CCNE se pose encore la question de l'usage d'un diagnostic préconceptionnel (DPC) dans des populations entières, afin de dépister certaines maladies comme cela a été le cas pour la thalassémie à Chypre ${ }^{6}$. En France, cela pourrait concerner la mucoviscidose. Le CCNE n'y est pas favorable, vu la gravité variable de cette maladie - il aurait aussi pu dénoncer une finalité préventive, voire eugéniste -, mais il ne se prononce pas sur le DPC en général. Les progrès dans le séquençage du génome rendant cette option relativement aisée, le CCNE est revenu sur le sujet dans son dernier avis, celui de 2018, dans lequel il propose que l'on autorise le DPC à toutes les personnes en âge de procréer qui le souhaitent [8]. Relevons que ce test est d'autant plus intéressant sur le plan éthique qu'il évite la destruction d'embryons, contrairement au DPI [11].

\footnotetext{
${ }^{5}$ Présence d'un chromosome surnuméraire à la $21^{\mathrm{e}}$ paire. Les personnes atteintes de trisomie 21 ont donc 47 chromosomes dans une cellule et non 46 .

${ }^{6}$ La campagne de dépistage des porteurs et d'éducation du public entreprise au début des années 1970 a
} permis de réduire fortement le nombre de nouveaux cas.
Retrouve-t-on les mêmes préoccupations en Belgique? Le Comité consultatif de bioéthique de Belgique (CCBB) a consacré, en 2009, un avis « relatif à l'utilisation du diagnostic génétique préimplantatoire pour détecter les porteurs sains d'une mutation causant une affection héréditaire grave qui peut entrâ̂ner un risque élevé pour les descendants ». Le sujet ne concerne pas d'abord des enfants pouvant souffrir d'une maladie grave, mais ceux qui sont porteurs d'un gène délétère et qui, eux-mêmes, ne seront pas malades (ce sont des porteurs sains). Ce cas pose au CCBB une difficulté juridique: «D'un point de vue médico-éthique, il semblait jusqu'ici généralement admis que le DPI n'est autorisé que s'il a une finalité proprement médicale, en ce sens qu'il a pour objectif de transférer un embryon sain pour éviter la naissance d'un enfant malade ou handicapé. Sur un plan strictement juridique, aucun des textes applicables ne permet de répondre de manière tranchée à la question de savoir si le recours au DPI est autorisé ou admissible, en vue d'éviter la naissance d'un enfant porteur sain d'une maladie génétique sévère » [12]. Toutefois, comme la loi belge autorise le DPI, y compris dans le cas du bébé-médicament, sauf s'il est de caractère eugéniste, c'est-à-dire s'il porte sur des caractères non pathologiques [13], il s'ensuit « que, dès lors que le diagnostic révélera quelque "imperfection" que ce soit, une sélection pourra s'ensuivre, puisque telle est la finalité de cette technique » [12]. Par conséquent, un DPI ayant pour but d'écarter un porteur sain est licite. Le CCBB examine notamment le cas de la mucoviscidose, une affection grave, pour laquelle le CCNE envisageait justement la possibilité d'un DPC.

En Suisse, le DPI a été examiné dans trois prises de positions par la Commission nationale d'éthique (CNE) [14-16]. Comme nous l'avons rappelé précédemment, jusqu'en 2016 le DPI était interdit, mais la majorité des membres du CNE a été, depuis le début, d'un avis différent en cas de pathologie grave, en excluant, toutefois, le cas des bébés-médicaments et des porteurs sains. En effet, le DPI permet d'éviter un DPN suivi d'un avortement, ce qui, d'une part, constitue un lourd fardeau pour le couple et, d'autre part, détruit un être humain (fœtus) à un stade de développement plus avancé. Pour la minorité, un des problèmes principaux du DPI est que la notion de maladie grave est floue, d'où le risque de pente glissante: «La définition pourrait, à terme, s'étendre à des défauts génétiques graves aux handicaps qu'il est possible de traiter, voire même aux dispositions corporelles contrevenant au sens esthétique du moment » [14]. La CNE est également très soucieuse d'éviter tout 
risque eugéniste, ce qu'elle comprend en un sens plus large que le CCNE et le CCBB, en le définissant comme «l'application de différents critères pour sélectionner certains êtres humains et en éliminer d'autres » [15], ce qui inclut un «eugénisme doux », «c'est-à-dire la discrimination des personnes handicapées via l'autorisation des procédés de sélection » [14]. On reconnaît ici l'argument expressiviste, et « une forme libérale d'eugénisme $»^{7}$, fondée sur l'autonomie des parents (leur droit à avoir un enfant en bonne santé), parfois plus apparente que réelle, vu la pression sociale.

\section{Les États généraux et au-delà}

Pendant les États généraux de la bioéthique, la question du DPI a été abordée, notamment lors des rencontres en régions. On observe que les inquiétudes des citoyens sont conformes à celles que les comités d'éthique avaient déjà formulées: "L'application des tests génétiques avant la naissance (DPN) ou chez l'embryon avant son transfert dans l'utérus (DPI) provoque, chez certains, la crainte d'un eugénisme et le sentiment que n'auraient le droit de vivre que ceux qui possèdent un "génome normal" » [3]. Eugénisme et discrimination rappelleraient donc l'argument expressiviste: la valeur des porteurs de génomes «anormaux » serait moindre et donc leur droit de vivre diminué. Quant aux critères d'application, les citoyens demeurent plutôt restrictifs, comme on le lit juste après : « Le principe d'un dépistage préconceptionnel, par exemple, de maladies familiales à transmission génétique ou néonatale des cinq maladies actuellement ciblées, est, dans certains débats, considéré comme utile », mais il ne serait pas judicieux d'étendre les indications, surtout pas à un séquençage massif, source de décisions épineuses, d'augmentation de la responsabilité et donc, d'anxiété - un effet latéral de la médecine prédictive. Comme le soulignait déjà le CCNE en 2009 (avis $n^{\circ} 107$ ), il existe un risque «de voir se multiplier des situations génératrices d'anxiété [...], du fait des progrès des techniques diagnostiques et de l'identification chez l'embryon à un stade précoce d'un grand nombre de marqueurs de susceptibilité à des maladies fréquentes » [7]. David Wasserman ${ }^{8}$ relève qu'avec la multiplication des tests prénatals, «les femmes désirant poursuivre leur grossesse auront le devoir de s'enquérir de l'actionabilité de la maladie de leur fœtus » [17], c'est-à-dire de l'existence ou non de possibilités d'interventions préventives ou thérapeutiques.

Toutefois, le Rapport souligne qu'il existe, dans la société, « une tension [...] entre la crainte d'un eugénisme et la volonté de réduire la souffrance, entre les risques et les dérives possibles de l'application élargie de dépistages et leurs bénéfices reconnus ou attendus $»^{9}$. L'eugénisme est régulièrement mentionné et, sur ce point, il serait utile de clarifier le concept, car celui dont il est ici question est bien différent de l'eugénisme politique classique (amélioration de la «race» des programmes du siècle dernier), ce dont les

${ }^{7}$ Le CCBB a aussi longuement analysé la notion d'eugénisme dans son avis $n^{\circ} 33$ [13] (chapitre 2) et en a présenté les différentes formes.

${ }^{8}$ Département de bioéthique, National Institutes of Health Clinical Center, Bethesda, Md., États-Unis.

${ }^{9}$ La question du bébé-médicament n'a pas été discutée ; elle n'est plus d'actualité. comités d'éthiques sont bien conscients, comme on I'a vu. Le Rapport demande d'ailleurs: «Le refus de l'eugénisme est un principe largement partagé, mais où commence l'eugénisme ? Vouloir un enfant en aussi bonne santé que possible en fait-il partie? » [18]. Une réponse négative s'impose évidemment dans ce cas, sinon, on devrait qualifier d'eugéniste la décision d'une femme d'avoir ses enfants avant l'âge de 25 ans dans le but de minimiser son risque de donner naissance à un enfant atteint de trisomie 21 . Mais la question de l'eugénisme déborde largement cette question et, en définitive, revient à demander s'il est légitime de poser des limites à la liberté procréative des parents et, si c'est le cas, quelles sont ces limites. Pour le critère de gravité, c'est une liste qui est proposée (cinq pathologies, dont la liste n'est d'ailleurs pas donnée dans le Rapport). Cela est en opposition aux avis des comités : «Le CCNE n'a pas soutenu l'idée de l'établissement d'une liste de maladies rendant acceptable le diagnostic anténatal. À supposer même qu'elle ne soit qu'indicative, une liste serait susceptible, à terme, de devenir normative. Elle substituerait une procédure standardisée à l'analyse au cas par cas », lit-on dans l'avis n 107 de 2009 [7]. En Suisse, la CNE s'oppose aussi aux listes dans sa prise de position de 2005 : «Trois raisons incitent à préférer une délimitation précise de la notion de "maladie grave" à une liste énonçant positivement ou négativement les maladies entrant en ligne de compte ou exclues pour un DPI. Une telle liste de maladies n'est pas souhaitable: (1) parce que la gravité d'une maladie, toute considération génétique et toute manifestation corporelle étant égales, dépend de la violence de la souffrance individuelle; (2) parce que le contexte génétique a une influence sur le degré de gravité de ses manifestations; (3) parce qu'une liste pourrait avoir des effets discriminatoires, aussi bien pour les personnes souffrant d'une maladie se trouvant sur la liste que pour celles dont ce ne serait pas le cas » [14]. Le comité belge a adopté une position analogue dans son avis de 2005 [13].

La difficulté de la question de la gravité est très souvent soulignée. Pour le CCNE (avis $n^{\circ} 107$ ), le concept est à la fois objectif et subjectif : «Bien qu'elle comporte une dimension objective irréductible, la notion de gravité admet également des degrés qui tiennent aux regards portés par la société, l'entourage proche, et les futurs parents » [7]. C'est qu'elle est liée à la souffrance, variable selon les situations et les personnes. $\varepsilon$ n outre, la souffrance prévisible de l'enfant se compose avec celle des parents. La CNE ne disait pas autre chose, et le CCBB approuve: "Il est impossible de donner une 
définition scientifique objective d'une maladie grave, puisque celle-ci dépend notamment du ressenti de la famille » [12]. Quelles maladies faut-il alors considérer comme graves ? Le CCNE en a rencontré quatre catégories dans la pratique:

«1. les anomalies du développement du fœtus décelées à l'échographie (anomalies des membres, nanisme, etc.)

2. les maladies génétiques à pénétrance élevée, ayant un retentissement sur la qualité de vie, parmi lesquelles certaines débutant précocement (myopathies, amyotrophies spinales, mucoviscidose), et d'autres se révélant plus tardivement (maladie de Huntington, certaines myopathies, etc.)

3. les maladies ayant un impact sur le développement psychomoteur, avec des répercussions sur la vie familiale et sociale (trisomie $21, \mathrm{X}$ fragile ${ }^{10}$, certaines hydrocéphalies)

4. I'existence d'un risque important d'une maladie mettant en jeu le pronostic vital chez des enfants ou des adultes jeunes (certains cancers héréditaires chez les enfants et adultes jeunes). »

Cette « liste » va bien au-delà de la mention des cinq maladies du Rapport et on a vu que le public n'était pas favorable à son extension. Les comités sont donc sur ce point plus libéraux que le public, ce qui se vérifie puisqu'une majorité des membres du CCNE s'est prononcée dès 2009 en faveur d'un élargissement du DPI sur un point précis: «Le Comité estime que l'interdiction de rechercher une trisomie 21 à l'occasion d'un DPI pour maladie génétique présente chez l'un des parents devrait être levée » [7], recommandation réitérée dans l'avis 129 [8]. En Suisse et en Belgique, cette recherche est déjà autorisée.

\section{Conclusion}

On observe donc en France un consensus large sur la question du DPI : il doit être réservé aux cas médicaux graves et a pour principale finalité d'éviter la venue au monde d'un enfant dont la qualité de vie sera très pauvre. S'il n'est pas exclu d'étendre les indications du diagnostic, ce n'est pas une urgence éthique. Depuis qu'elle a autorisé le DPI, la Suisse a adopté une position assez proche de celle de la France et est même devenue un peu plus libérale, puisqu'elle ne limite pas le test à une seule pathologie. C'est la Belgique qui va le plus loin, en autorisant le diagnostic dès que la santé est en jeu, donc également sur des embryons qui ne souffriront pas eux-mêmes d'une maladie, mais sont porteurs de gènes délétères.

Dans un futur proche, deux tâches paraissent s'imposer. La première est pédagogique. Elle consiste, nonobstant les efforts déjà faits, à clarifier encore l'usage de l'objection d'eugénisme. Peut-être, d'ailleurs, serait-il souhaitable de ne pas parler d'eugénisme dans la question du DPI, car cette expression emporte avec elle un jugement de valeur fortement négatif qui fait obstacle à une discussion argumentée des torts et des bienfaits liés à cette pratique. La seconde est sociétale : il

${ }^{10}$ Cette maladie génétique héréditaire est liée à une mutation du gène FMRI (fragile $X$ mental retardation 1) dont la fonction est encore mal connue. Ce gène est porté par le chromosome $X$. Cette affection touche environ 1/4 000 garçons et 1/8 000 filles. La mutation consiste en la répétition anormale d'une partie du gène. est nécessaire de réfléchir sur la question de la gravité et de bien évaluer ce qui doit compter moralement, tant de son côté objectif que de son côté subjectif. À un moment où la médecine prédictive étend son emprise et où le séquençage du génome devient routinier, il n'est pas bon d'attendre plus longtemps. $\diamond$

\section{SUMMARY}

Preimplantation genetic diagnosis (PGD) in the era of predictive medicine

High speed sequencing is revolutionizing the various genetic tests and in particular preimplantation genetic diagnosis (PGD), opening the doors to an increasingly efficient predictive medicine. PGD itself is not new, and the various legistations have been dealing with it for some twenty years. National ethics committees, for their part, have widely debated antenatal tests in the context of medically assisted procreation. This paper examines the work of these Committees on PGD in three States, France, Belgium and Switzerland, in order to highlight their similarities and differences. The subject has also been raised and discussed in the context of the États généraux de la bioéthique which took place this year in France and, given the progresses made recently, they have echoed certain demands of civil society. However, in France, caution continues to dominate. $\diamond$

\section{REMERCIEMENTS}

Je remercie Pierre Le Coz (ancien membre du CCNE), Marie-Geneviève Pinsart (vice-présidente du CCBB belge) et Simone Romagnoli (collaborateur scientifique au CNE suisse) d'avoir relu une première version de ce texte. Leurs commentaires m'ont été très utiles.

\section{LIENS D'INTÉRÊT}

L'auteur déclare n'avoir aucun lien d'intérêt concernant les données publiées dans cet article.

\section{RÉFÉRENCES}

1. Lipworth W, Mason P, Kerridge I, loannidis J. Ethics and epistemology in big data research. Bioethical Inquiry 2017 ; 14 : 489-500.

2. Comité Consultatif National d'Éthique pour les sciences de la vie et de la santé. Avis sur les problèmes posés par le diagnostic prénatal et périnatal. Avis n $5,1985,14 \mathrm{p}$.

3. Rapport de synthèse du Comité Consultatif National d'Éthique. Les Ulis : EDP Sciences, 2018 : 36-7.

4. Étude de législation comparée $n^{\circ} 188$ : le diagnostic préimplantatoire. Octobre 2008. https ://www.senat.fr/lc/lc188/lc1880.html.

5. Conseil fédéral. Autoriser le diagnostic préimplantatoire en Suisse à des conditions strictes. Communiqué du 11 avril 2016. https://www.admin. $\mathrm{ch} / \mathrm{gov} / \mathrm{fr} /$ accueil/documentation/communiques/communiques-conseilfederal.msg-id-61294.html

6. Comité consultatif national d'éthique pour les sciences de la vie et de la santé. Réflexions sur l'extension du diagnostic préimplantatoire. Avis $\mathrm{n}^{\circ} 72,2002,18 \mathrm{p}$.

7. Comité Consultatif National d'éthique pour les sciences de la vie et de la santé. Avis sur les problèmes éthiques liés aux diagnostics anténatals. Avis $n^{\circ} 107,2009,28 \mathrm{p}$. 


\section{RÉFÉRENCES}

8. Comité Consultatif National d'éthique pour les sciences de la vie et de la santé. Contribution du Comité consultatif national d'éthique à la révision de la loi de bioéthique 2018-2019. Avis $n^{\circ} 129,2018,160 p$

9. Gosseries A. Penser la justice entre les générations. Paris : Aubier, 2004 : chap. 1.

10. Wilkinson S. Choosing Tomorrow's Children. Oxford : Oxford UP, 2010 : chap. 6.

11. Vialard F, Molina-Gomes D, Hammoud I et al. Analyse du premier globule polaire et diagnostic préconceptionnel : état des lieux. In : Poncelet C, Sifer C, eds. Physiologie, pathologie et thérapie de la reproduction chez l'humain. Paris: Springer, 2011 : 389-99.

12. Comité Consultatif de Bioéthique de Belgique. Avis relatif à l'utilisation du diagnostic génétique préimplantatoire pour détecter les porteurs sains d'une mutation causant une affection héréditaire grave qui peut entraîner un risque élevé pour les descendants. Avis $n^{\circ} 49,2009,28 p$

13. Comité Consultatif de Bioéthique de Belgique. Avis relatif aux modifications géniques somatiques et germinales à visées thérapeutiques et/ou mélioratives. Avis $n^{\circ}$ 33, 2005, $52 \mathrm{p}$
14. Commission Nationale d'Éthique. Diagnostic préimplantatoire. Prises de position $\mathrm{n}^{\circ} 10,2005,54 \mathrm{p}$.

15. Commission Nationale d'Éthique. Diagnostic préimplantatoire I/. Prises de position $n^{\circ} 14,2007,24 p$.

16. Commission Nationale d'Éthique. La procréation médicalement assistée. Prises de position $n^{\circ} 22,2013,59 \mathrm{p}$.

17. Wasserman D. Fetal medicine and the pregnant woman. Hastings Center Report $2018 ; 48(2)$ : page de garde.

18. Baertschi B. De l'humain augmenté au posthumain, chap. 7. Paris : Vrin, 2019 (sous presse)

ertrand Jordan a participé à l'extraordinaire aventure de la biologie moléculaire, encore balbutiante dans les années 1960, mais qui a complètement révolutionné les sciences du vivant au cours des décennies suivantes.

L'ADN, quasiment inaccessible au début de cette période, intervient maintenant dans d'innombrables recherches, de l'écologie à l'anthropologie, sans oublier bien sûr la médecine dont il fait désormais partie intégrante. Nous pouvons aujourd'hui lire intégralement

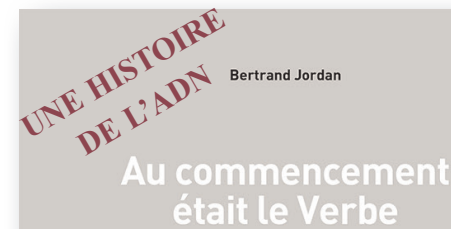

Une histoire personnelle de l'ADN

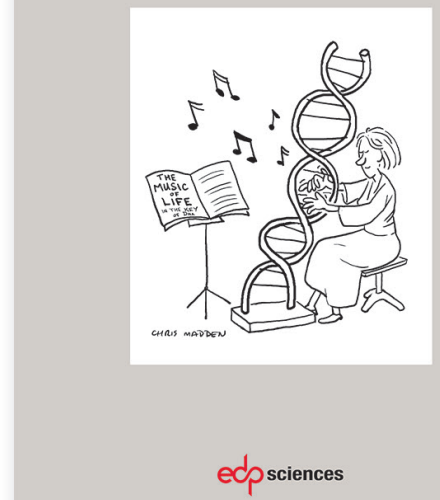

edpsciences

I'ADN d'une personne en quelques heures, et en tirer de précieuses informations pour la prévention et le traitement de nombreuses maladies - et nous n'en sommes qu'au début de cette nouvelle médecine !

Acteur mais aussi témoin de ces avancées, Bertrand Jordan fait ici un récit très personnel et sans langue de bois de sa vie avec l'ADN. Ce livre ne prétend pas être une histoire complète de la biologie moléculaire, mais il illustre son développement, révélant parfois le «dessous des cartes » grâce aux expériences vécues par son auteur.

TIRÉS À PART

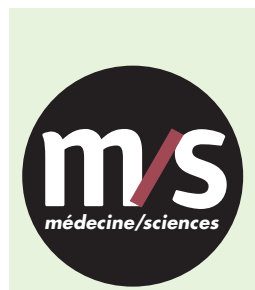

Tarifs d'abonnement $\mathrm{m} / \mathrm{s}-2019$

Abonnez-vous

à médecine/sciences
$>$ Grâce à $\mathrm{m} / \mathrm{s}$, vivez en direct les progrès des sciences biologiques et médicales

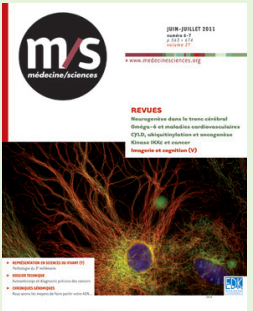

